\title{
Analysis of Chemokines and Receptors Expression Profile in the Myelin Mutant Taiep Rat
}

\author{
Guadalupe Soto-Rodriguez, ${ }^{1}$ Juan-Antonio Gonzalez-Barrios, ${ }^{2}$ \\ Daniel Martinez-Fong, ${ }^{3}$ Victor-Manuel Blanco-Alvarez, ${ }^{1}$ Jose R. Eguibar, ${ }^{4}$ Araceli Ugarte, ${ }^{4}$ \\ Francisco Martinez-Perez, ${ }^{5}$ Eduardo Brambila, ${ }^{1}$ Lourdes Millán-Perez Peña, ${ }^{1}$ \\ Nidia-Gary Pazos-Salazar, ${ }^{1}$ Maricela Torres-Soto, ${ }^{1}$ Guadalupe Garcia-Robles, ${ }^{1}$ \\ Constantino Tomas-Sanchez, ${ }^{1}$ and Bertha Alicia Leon-Chavez ${ }^{1}$ \\ ${ }^{1}$ Facultad de Ciencias Químicas, Benemérita Universidad Autónoma de Puebla, 14 Sur y Avenida San Claudio, \\ 72570 Puebla, PUE, Mexico \\ ${ }^{2}$ Laboratorio de Medicina Genómica, Hospital Regional $1^{\circ}$ de Octubre, ISSSTE, Avenida Instituto Politécnico Nacional No. 1669, \\ 07760 México, DF, Mexico \\ ${ }^{3}$ Departamento de Fisiología, Biofísica y Neurociencias, Centro de Investigación y de Estudios Avanzados del \\ Instituto Politécnico Nacional, Apartado Postal 14-740, 07000 México, DF, Mexico \\ ${ }^{4}$ Instituto de Fisiología, Benemérita Universidad Autónoma de Puebla, 14 Sur 6301, 72560 Puebla, PUE, Mexico \\ ${ }^{5}$ Laboratorio de Genómica de Celomados, Grupo de Microbiología y Genética de la Escuela de Biología, \\ Universidad Industrial de Santander, Apartado Postal 680002, Bucaramanga, Colombia
}

Correspondence should be addressed to Bertha Alicia Leon-Chavez; alileonch@gmail.com

Received 21 December 2014; Revised 8 March 2015; Accepted 9 March 2015

Academic Editor: Cinzia Signorini

Copyright ( $) 2015$ Guadalupe Soto-Rodriguez et al. This is an open access article distributed under the Creative Commons Attribution License, which permits unrestricted use, distribution, and reproduction in any medium, provided the original work is properly cited.

\begin{abstract}
Taiep rat has a failure in myelination and remyelination processes leading to a state of hypomyelination throughout its life. Chemokines, which are known to play a role in inflammation, are also involved in the remyelination process. We aimed to demonstrate that remyelination-stimulating factors are altered in the brainstem of 1- and 6-month-old taiep rats. We used a Rat $\mathrm{RT}^{2}$ Profiler PCR Array to assess mRNA expression of 84 genes coding for cytokines, chemokines, and their receptors. We also evaluated protein levels of CCL2, CCR1, CCR2, CCL5, CCR5, CCR8, CXCL1, CXCR2, CXCR4, FGF2, and VEGFA by ELISA. Sprague-Dawley rats were used as a control. PCR Array procedure showed that proinflammatory cytokines were not upregulated in the taiep rat. In contrast, some mRNA levels of beta and alpha chemokines were upregulated in 1-month-old rats, but CXCR4 was downregulated at their 6 months of age. ELISA results showed that CXCL1, CCL2, CCR2, CCR5, CCR8, and CXCR4 protein levels were decreased in brainstem at the age of 6 months. These results suggest the presence of a chronic neuroinflammation process with deficiency of remyelination-stimulating factors (CXCL1, CXCR2, and CXCR4), which might account for the demyelination in the taiep rat.
\end{abstract}

\section{Introduction}

The taiep rats exhibit hypomyelination and suffer progressive demyelination resulting in a highly hypomyelinated central nervous system (CNS) as they reach adulthood $[1,2]$. This demyelinating process has been associated with nitrosative stress in vivo and in vitro $[3,4]$, glial-microglial activation and lymphocyte migration [4], and an increase in lipoperoxidation, caspase-3 activation, and cell death in vivo [5]. The taiep rat has been considered as a chronic animal model of multiple sclerosis (MS) [6], which is characterized by a remyelination failure in areas of chronic demyelination with absence of oligodendrocyte progenitor cells (OPCs) and acute inflammation [7]. It has been suggested that astrogliosis 
in a microenvironment of chronic inflammation forms a barrier that impedes the OPC migration [7]. However, induction of an acute inflammation in areas of chronic demyelination can activate remyelination as proven in the taiep rat [8]. These results suggest that OPCs in the taiep rat are unable to generate myelinating oligodendrocytes due to the lack of the stimulatory factors and/or the presence of inhibitory factors [8].

Previous studies in the taiep rat have shown glial cell priming [3], reactive astrogliosis since 1 month of age [9], and lymphocyte infiltration at 6 months of age [4], suggesting the participation of glial cells in the early age and leukocytes in the adulthood. To date, the immunological mediators involved in those differential cell processes in the taiep rat remain unknown. Several reports in different animal models [10-15] and in human patients with MS [16] sustain the involvement of chemokines and their receptors in the CNS inflammation. Alternatively, chemokines also participate in myelin development. CXCL1 has been shown to play an important role in proliferation, differentiation, migration, and maturation of oligodendroglial cells and myelin synthesis [17-19]. Moreover, the deficit of CXCL1 and CXCR2 causes failure in the myelination process due to an aberrant migration of OPCs in the spinal cord white matter [20]. CXCL12 activating CXCR4 receptor has been involved in the OPCs maturation and remyelination failure [21]. CXCL12 also acts as a growth factor for stimulating the astrocyte proliferation and neuronal cells $[22,23]$, via extracellular signal-regulated kinases (ERK 1/2) [24].

Based on the finding that the remyelination process is absent in the taiep rat $[7,8]$, it is important to determine chemokines and their receptors especially those that act as remyelination-stimulating factors. The present work aimed to evaluate whether a deregulation of chemokine expression profile occurs in the taiep rat. We used $\mathrm{RT}^{2}$ Gene Profiler PCR Array to assess cytokines, chemokines, and their receptors and ELISA to measure CCL2, CCR2, CCL5, CCR5, CXCL1, CXCR2, and CXCR4. The measurements were made in the brainstem from 1- to 6-month-old taiep rats because this is the second region most affected and presents atypical cells at those ages [4]. The results were compared with those in Sprague-Dawley (SD) rats. Our results showed the deficiency of CXCL1 and CXCR4 levels in the taiep rats, suggesting that these chemokines might be involved in the remyelination failure in those animals.

\section{Materials and Methods}

2.1. Experimental Animals. Taiep rats of different ages (one and six months old) were obtained from the vivarium of the Institute of Physiology, BUAP. Sprague-Dawley (SD) rats (negative controls) were supplied by CINVESTAV vivarium. Institutional Animal Care and Use Committee (IACUC) approved our animal use procedures with the protocol number 410-08. Animals were maintained in rooms with controlled conditions of temperature $\left(22 \pm 1^{\circ} \mathrm{C}\right)$ and lightdark cycle (12:12 h light: dark; light onset at 07:00). Food and water were provided ad libitum. All procedures were in accordance with the Mexican current legislation, the NOM062-ZOO-1999 (SAGARPA), based on the Guide for the Care and Use of Laboratory Animals, NRC. All efforts were made to minimize animal suffering.

2.2. PCR Array. The total RNA $(1.0 \mu \mathrm{g})$ extracted from 1- to 6-month-old brainstem of SD and taiep rats was quantified with a NanoDrop Spectrophotometer (Thermo Scientific NanoDrop Technologies, Wilmington, DE, USA). Reverse transcription reaction was made with the $\mathrm{RT}^{2} \mathrm{PCR}$ Array First Strand Kit from SABiosciences (Qiagen Company). Real-time PCR was conducted on a 384-well plate for Chemokines and Receptors $\mathrm{RT}^{2}$ Profiler PCR Array of Rat (PARN-022Z, Qiagen), which contains a profile for the expression of 84 genes that encode chemokines and their receptors and cytokines. The amplification assays were made using a 7900HT Fast Real-Time PCR System (Applied Biosystems, Foster City, CA, USA) (http://www.sabiosciences .com/PCRArrayPlate.php).

2.3. Enzyme-Linked Immunosorbent Assay (ELISA). ELISA was used to determine the protein levels of CCL2, CCR1, CCR2, CCL5, CCR5, CCR8, CXCL1, CXCR2, CXCR4, FGF2, and VEGFA in homogenates obtained from the brainstem of SD or taiep rats at 1 and 6 months of age $(n=5$ per age in each group), as described previously [4]. The protein content was determined using the method by Sedmak and Grossberg [25]. Aliquots containing $5 \mu \mathrm{g}$ of total protein were placed into wells of ELISA plates to determine chemokines and receptors in separate assay. Then, proteins were denatured by the addition of $100 \mu \mathrm{L}$ of $0.1 \mathrm{M}$ carbonate buffer ( $\mathrm{pH}$ 8.0) added into each well and the plate was incubated for $18 \mathrm{~h}$ at $4^{\circ} \mathrm{C}$. To block nonspecific binding sites, $200 \mu \mathrm{L}$ of $0.5 \%$ bovine serum albumin, IgG-free, was added to each well at room temperature (RT). After $30 \mathrm{~min}$ of incubation, the wells were washed thrice with PBS-Tween $20(0.1 \%)$. Rabbit monoclonal antibodies to CCL2, CCR1, CCR2, CCL5, CCR5, CCR8, CXCL1, CXCR2, CXCR4, FGF2, and VEGFA (1:500 dilution, Abcam Inc., Cambridge, MA, USA) detect both native and denatured proteins. They were added into each well and incubated for $2 \mathrm{~h}$ at RT. After three washings with PBS, a horseradish peroxidase-conjugated goat anti-rabbit IgG (1:1000 dilution, Dako North America Inc., Carpinteria, CA, USA) was added and incubated for $2 \mathrm{~h}$ at RT. The antigen-antibody complex was revealed by adding $100 \mu \mathrm{L}$ of 2,2' -azino-bis(3-ethylbenzthiazoline6-sulfonic acid) (ABTS) containing $0.3 \% \mathrm{H}_{2} \mathrm{O}_{2}$ into each well. After $15 \mathrm{~min}$, the optical density (OD) was determined at $415 \mathrm{~nm}$ using a Benchmark multiplate reader (Bio-Rad, Hercules, CA, USA) as was described elsewhere [4].

2.4. Indirect Immunofluorescence. CCR2, CCR5, CXCR2, and CXCR4 were detected in brain sagittal slices of 6-monthold $\mathrm{SD}$ and taiep rats ( $n=3$ per each group). Rats were deeply anesthetized with chloral hydrate and perfused through the ascending aorta with $100 \mathrm{~mL}$ of PBS $1 \mathrm{x}$ and then by $150 \mathrm{~mL}$ of $4 \%$ paraformaldehyde in PBS. Their brains were removed and maintained in the fixative for $48 \mathrm{~h}$ 


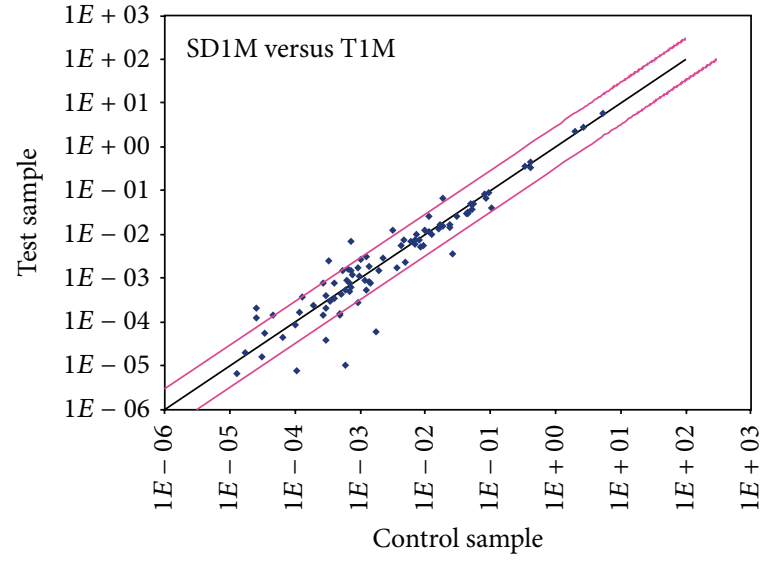

(a)

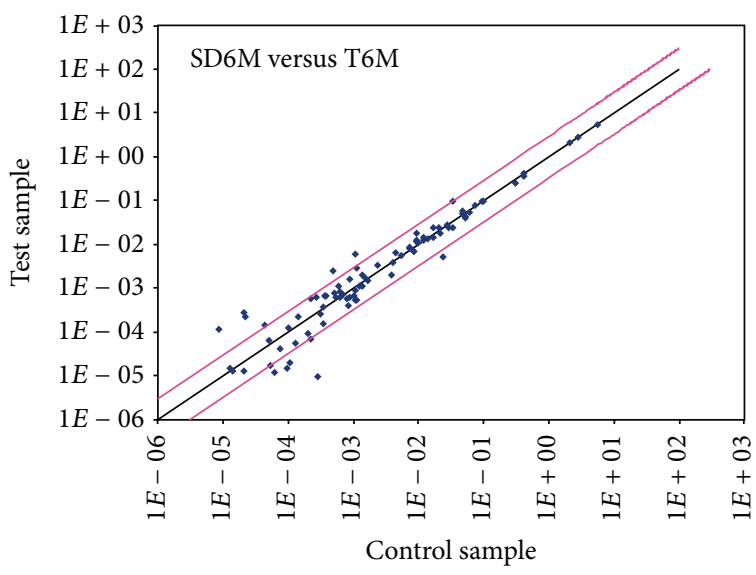

(b)

Figure 1: Overview of scatter plot of expression of 84 genes. Analysis of expression of genes from brainstem of taiep rats compared with Sprague-Dawley rats by RT ${ }^{2}$ Profiler PCR Array. The black line indicates fold changes $\left[2^{\left(-\Delta C_{t}\right)}\right]$ of 1 . The pink lines indicate the desired fold change in gene expression threshold, defined by the user with the entry in cell A1. SD1M = 1-month-old Sprague-Dawley $(\mathrm{SD})$ rats; T1M = 1-month-old taiep rats; SD6M = 6-month-old SD rats; $\mathrm{T} 6 \mathrm{M}=6$-month-old taiep rats.

at $4^{\circ} \mathrm{C}$. Each brain was included in paraffin and was cut into $3 \mu \mathrm{m}$ slices on the sagittal plane using a Leica RM 2135 microtome (Leica Microsystems, Nussloch, Germany). Slices were individually collected on a glass slide. Tissue slices (previously deparaffinized) were rehydrated and incubated with $0.5 \%$ IgG-free bovine serum albumin in PBS-Tween 20 $(0.1 \%)$ for $20 \mathrm{~min}$ at RT. Slices were incubated with rabbit monoclonal antibodies to CCR2, CCR5, CXCR2, or CXCR4 (1:500 dilution, Abcam Inc., Cambridge, MA, USA) at $4^{\circ} \mathrm{C}$ overnight. The secondary antibody was a goat anti-rabbit IgG conjugated with fluorescein isothiocyanate (FITC). The counterstaining was made using DAPI or propidium iodide $(2 \mu \mathrm{g} / \mathrm{mL})$. Tissue slices were mounted on glass slides using VECTASHIELD (Vector Laboratories, Burlington, Ontario, Canada). The fluorescence within the cells was analyzed with $5 \mathrm{x}$ and $40 \mathrm{x}$ objectives of a Leica DMIRE2 microscope using the filters A for DAPI, K3 for FITC, and TX2 for propidium iodide (Leica Microsystems, Wetzlar, Germany). The images were digitalized with a Leica DC300F camera (Leica Microsystems, Nußloch, Germany) and processed with a workstation Leica FW4000, version V1.2.1 (Leica Microsystems Vertrieb GmbH, Bensheim, Germany). Samples incubated with only the secondary antibodies were used as negative controls.

2.5. Statistical Analysis. All values are provided as the mean \pm standard error of the mean (SEM). The difference between the groups with respect to age in all the assays was analyzed using a one-way ANOVA test. The differences between SD and taiep rats were determined with unpaired Student's $t$ test. All statistical analyses were performed using the GraphPad Prism software (GraphPad Software Inc., San Diego, CA, USA). $P$ values $<0.05$ were considered significant. Analysis of qPCR data was performed based on a webbased PCR Array Data Analysis protocol (http://pcrdataanalysis.sabiosciences.com/pcr/arrayanalysis.php) provided by SABiosciences (Qiagen) and the results are expressed as a fold change.

\section{Results}

The scatter plots show the expression profile of 84 genes of taiep rat in comparison with the control profile of SD rats (Figure 1). No upregulation of proinflammatory cytokines was determined at any age and group studied. In contrast, downregulation was established for IL-1 $\beta(-3.3, P<0.05)$ and IL-10 $(-8.0, P<0.05)$ at 1 -month-old, ITG- $\beta 2$ at both 1 - $(-59.3, P=0.01)$ and 6-month-old $(-5.4, P=$ $0.01)$, and MAPK1 at 6-month-old $(-29.9, P=0.01)$ taiep rats (Figure 2). Downregulation was also established for inflammation mediators such as formyl peptide receptor 1 (FPR1) at $1-(-13.5, P<0.05)$ and at 6-month-old $(-5.31$, $P<0.05)$ and GPR17 at $1-(-7.03, P=0.05)$ and at 6 -monthold $(-4.6, P<0.05)$ taiep rats. These results show that taiep rat brainstem exhibits a decrease in inflammatory and antiinflammatory cytokine.

Expression of some chemokines and their receptors was modified in the brainstem of taiep rat when compared with those in SD rat. Gene upregulation of CCL19 at 1(3.5, $P=0.01)$ and at 6-month-old (3.0, $P=0.05)$, CCL22 at 1-month-old (2.8, $P=0.005)$, CCL4 at 1-month-old (9.3, $P=0.003)$, CCL5 at $1-(7.4, P=0.02)$ and at 6 -month-old (4.8, $P=0.05)$, and CCR6 at 1-month-old (2.4, $P=0.05)$ taiep rats was found. Alpha chemokines were also upregulated; CXCL10 at $1-(1.9, P=0.02)$ and at 6-month-old $(1.8, P=$ $0.004)$, CXCL11 at 1-month-old (2.6, $P=0.003)$, and VEGFA at $1-(4.8, P<0.05)$ and at 6 -month-old $(10, P=0.03)$ (Figure 2) taiep rats were found.

CCR1L1 was downregulated at 1-month-old $(-2.36, P=$ $0.02)$, CCR8 at 1 -month-old $(-6.6, P=0.03)$, and CXCR4 at 1-month-old (-28.8, $P<0.05)$, at 6-month-old $(-5.3, P<$ $0.05)$, and at 6 -month-old $(-2.2, P=0.01)$ taiep rats. 


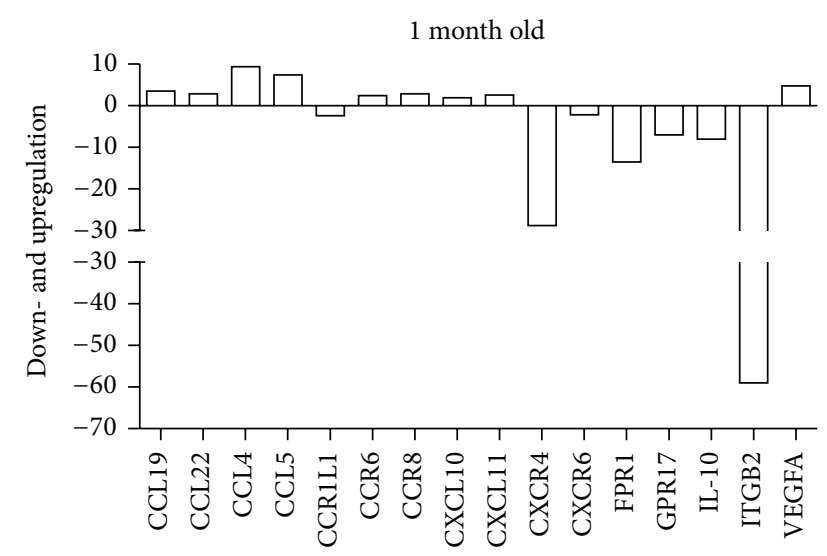

(a)

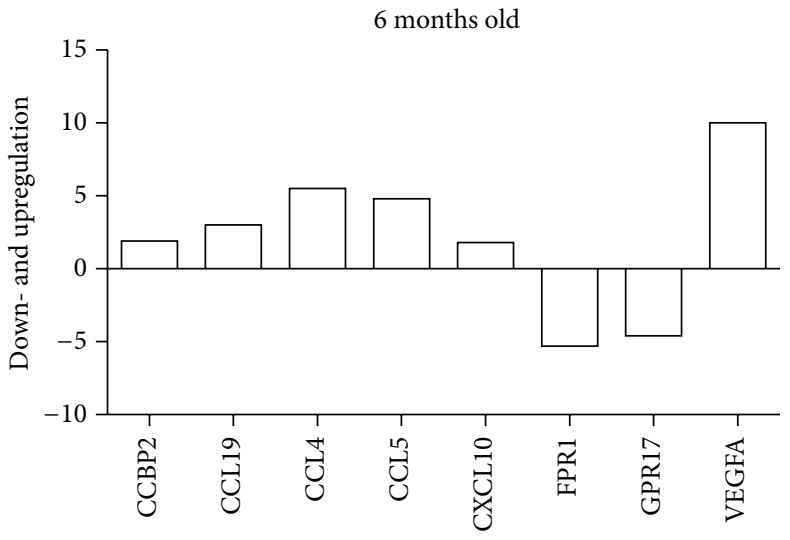

(b)

FIgURE 2: Chemokine expression profile in the brainstem of taiep rats 1 and 6 months old. Deregulation in chemokines and chemokine receptors was determined by $\mathrm{RT}^{2}$ Profiler PCR Array in the brainstem of taiep rats one and six months old. Values show fold up- or downregulation as compared to control group, Sprague-Dawley rats at the same age.

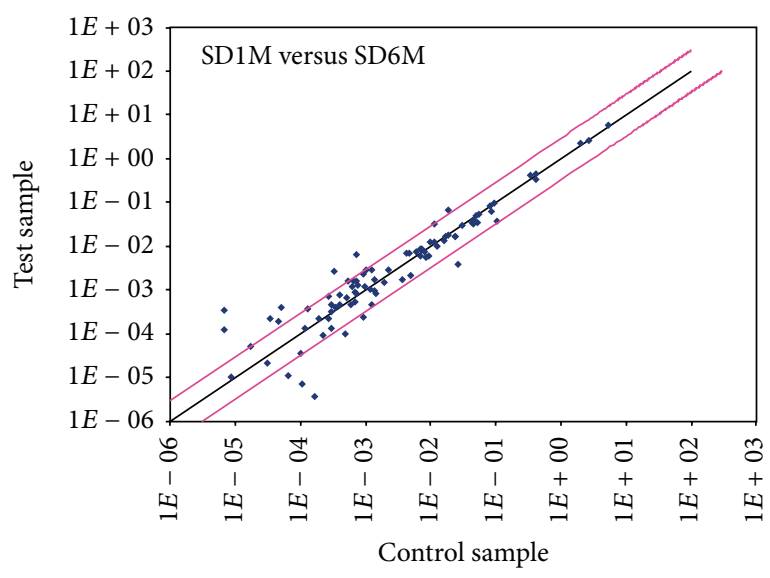

(a)

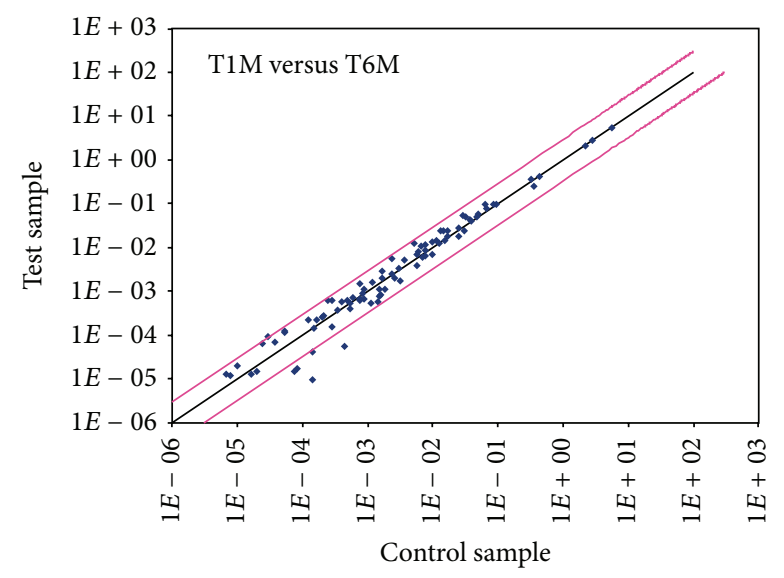

(b)

FIGURE 3: Overview of scatter plot on expression of 84 genes. Analysis of gene expression in the brainstem at different ages of Sprague-Dawley and taiep rats by $\mathrm{RT}^{2}$ Profiler PCR Array. The black line indicates fold changes $\left[2^{\left(-\Delta C_{t}\right)}\right]$ of 1 . The pink lines indicate the desired fold change in gene expression threshold, defined by the user with the entry in cell A1. SD1M = 1-month-old Sprague-Dawley (SD) rats; T1M = 1-month-old taiep rats; $\mathrm{SD} 6 \mathrm{M}=6$-month-old $\mathrm{SD}$ rats; $\mathrm{T} 6 \mathrm{M}=6$-month-old taiep rats.

Comparisons between results from 6-month-old versus 1month-old rats in the same group show no significant changes in gene expression in SD rats, except for two downregulated chemokines, CXCL9 $(-3.9, P<0.05)$ and TGFB1 $(-3.6, P<$ $0.05)$, while, in taiep rats, gene expression was downregulated for 5 chemokines: CCR8 $(-5.2, P=0.05)$, CCR9 $(-7.7, P<$ $0.05)$, CXCL5 $(-2.2, P<0.05)$, MAPK1 $(-15.3, P<0.05)$, and TGFB1 $(-3.5, P<0.05)$ (Figure 3$)$.

Comparison of protein levels between taiep rats and SD rats showed changes in chemokine receptors and growth factors at both ages. At 1 month of age, receptors that increased were CCR2 $(43.5 \pm 5.1 \%)$, CCR8 (111.3 $\pm 12.4 \%)$, CXCR4 $(115.8 \pm 18.1 \%)$, and FGF2 $(27.6 \% \pm 4.1)$. In contrast, CCR5 $(-19.8 \pm 5.1)$ and CXCL1 $(-82.2 \pm 1.6 \%)$ were decreased (Figure 4$)$. In the brainstem of 6 -month-old rats, protein levels were decreased for CCL2 $(-39.0 \% \pm 5.5 \%)$, CXCL1 $(-80.9 \% \pm 3.6 \%)$, CCR1 $(-14.4 \% \pm 7.9 \%)$, CCR2
$(-41.2 \% \pm 1.4 \%)$, CCR5 $(-21.8 \% \pm 5.8 \%)$, CCR8 $(-34.6 \% \pm$ $5.8 \%)$, CXCR4 ( $-54.4 \% \pm 3.7 \%)$, and, the growth factor, FGF2 $(-42.7 \% \pm 2.7 \%)$. In contrast, VEGFA $(125.5 \% \pm 29.7 \%)$ levels increased (Figure 4).

Immunofluorescence studies showed decreased intensity against CCR5 and CXCR4 in the brainstem of 6-month-old taiep rats in comparison with SD rats. CCR5 immunostaining was evident in glial cells located in the white matter of both $\mathrm{SD}$ and taiep rats. CCR2 and CXCR2 were found in glial cells and neurons, but CXCR4 was observed in glial cells (Figure 5). CXCR2 immunofluorescence intensity in taiep rats was not different from that in SD rats (Figure 5).

\section{Discussion}

Results obtained in this work support the idea that the taiep rat exhibits a chronic neuroinflammatory profile that 


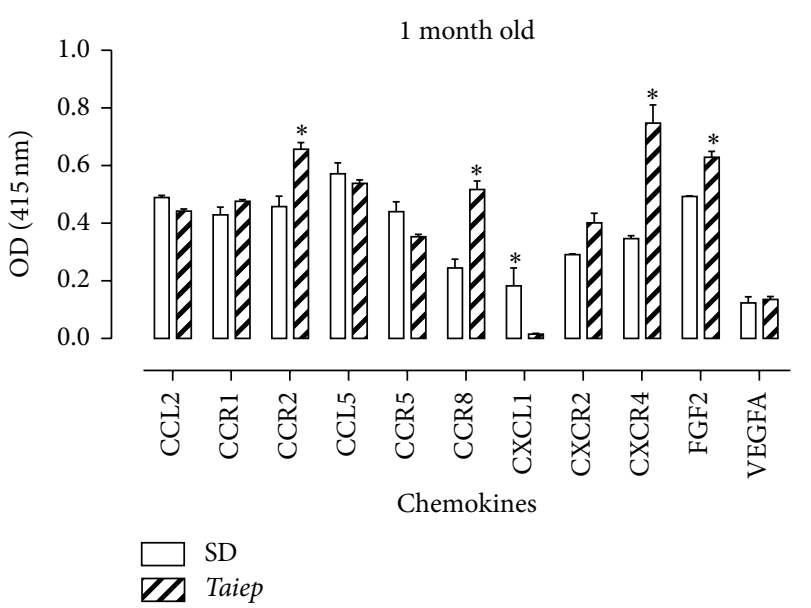

(a)

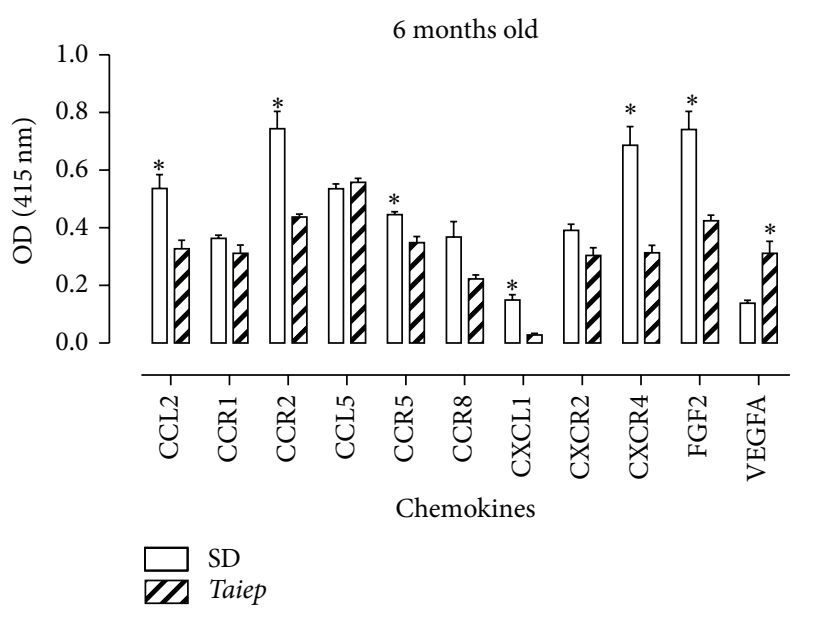

(b)

FIGURE 4: Protein levels of chemokines and their receptors in the brainstem of Sprague-Dawley (SD) and taiep rats. Chemokines, receptors, and growth factors were assayed using indirect ELISA. Each value represents the mean \pm SEM of 5 independent experiments made in triplicate.

*Significantly different from SD rats (Student's $t$-test). The significance was established at $P<0.05$.
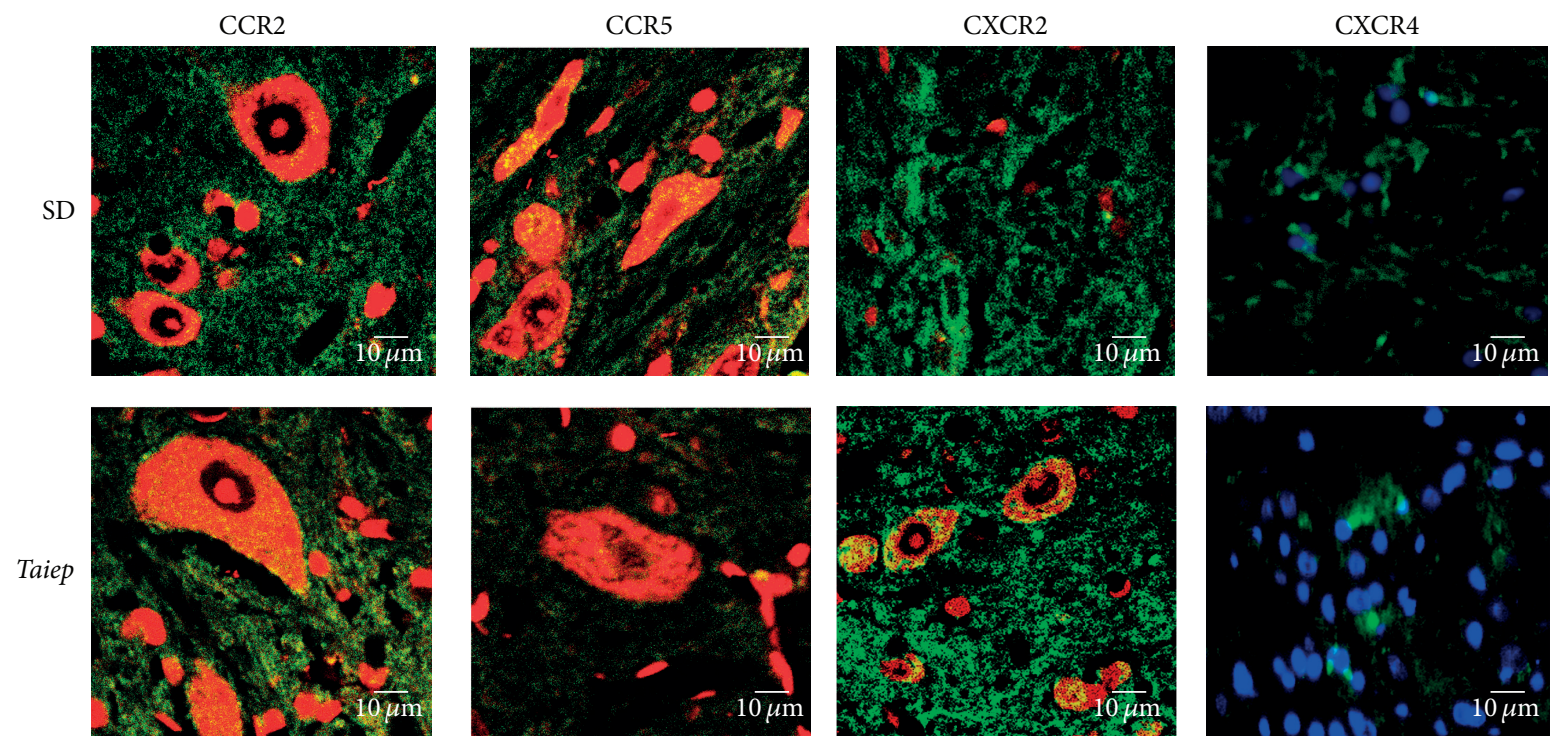

FIGURE 5: Immunoreactivity against receptors in the brainstem of taiep and Sprague-Dawley rats 6 months old. Paraffin-included slices of $3 \mu \mathrm{m}$ were immunostained with rabbit monoclonal anti-CCR2, anti-CCR5, anti-CXCR2, and anti-CXCR4 (fluorescein, green). Propidium iodide (red) or DAPI (blue) was used as nuclear counterstaining. SD; Sprague-Dawley rats.

is different from other models of demyelination such as EAE. The major differences of taiep rats in comparison with EAE are that cytokines such as IFN- $\gamma$, TNF- $\alpha$, and IL-6 were unaltered but IL-1 $\beta$ and IL-10 were downregulated in 1-month-old taiep rats. In normal pups, the exposure to stress stimuli leads to decreased levels of IL-10 and IL-1 $\beta$ in serum [26]. Downregulation of IL-1 $\beta$ and IL-10 in the brainstem of taiep rats might be explained in part by the motor alterations of taiep mother and pups, acting as a stress stimulus. In addition, downregulation of TGF- $\beta 1$ was found in taiep rats. As TGF- $\beta 1$ suppresses inflammation and promotes neuronal survival in adult CNS, the lack of these effects could be associated with the increased neuronal apoptosis and necrosis in the brain of adult taiep rats [5]. The decrease in TGF- $\beta 1$ expression at 6-month-old taiep and SD rats might also be a sign of CNS aging.

We found that GPR17 was downregulated in the taiep rats. This $\mathrm{G}$ coupled protein receptor is an important mediator of OPC differentiation and white matter repair that is expressed by a subset of OPCs to operate as an early sensor of brain damage [27]. Accordingly, inhibition of GPR17 expression causes impairment in oligodendrocytes differentiation and myelination in in vivo and in vitro systems [28]. Supporting this, GPR17 downregulation could contribute to alterations in remyelination in the taiep rats. Another coupled protein receptor that was downregulated in the brainstem of 
1-month-old taiep rats was FPR1 (formyl peptide receptor 1), which mediates the chemotaxis, activation, and cytokine gene transcription in phagocytic leucocytes in response to bacterial formylated chemotactic peptides [29]. These findings suggest that the immune response is decreased in the taiep rat, producing a predominant chronic neuroinflammatory process or astrocyte priming as was reported previously in cell culture [3].

CCL19 is a chemokine involved in the maintenance of chronic neuroinflammation [30, 31]. Accordingly, this chemokine was upregulated in the brainstem of taiep rats. The CCR8 mRNA and protein were also upregulated at the age of 1 month in the taiep rats. This finding can explain the accumulation of microglial cells in the CNS of taiep rats reported previously [4] due to the action of promoting chemotaxis of mononuclear cells and phagocytosis [32]. However, CCR8 protein levels were decreased in 6-monthold taiep rats, suggesting decrease in the infiltration of macrophages.

Although CCL5 was upregulated in the taiep rats at the age groups studied, protein levels were decreased in the brainstem, as well as its receptor CCR5 in 6-month-old rats. The reduction of CCR5 in mice prevents macrophage infiltration and demyelination $[33,34]$ and lack of CCL5 decreases neuronal survival $[33,35]$. The decrease in CCL5 and CCR5 supports the cell death that occurs in taiep rats [5].

CXCL10 is involved in the T cell trafficking in the MS [36, 37]. Accordingly, upregulation of CXCL10 might explain the presence of CD $4(+)$ cells in the CNS of taiep rats reported previously [4].

The deficiency of the CXCL1 and CXCR4 proteins in the taiep rats might affect the proliferative response and recruitment of OPC to the injured areas $[21,38]$. Lack of CXCL1 may also contribute to differentiation failure of OPCs $[39,40]$. Altogether, these alterations of OPCs limit the remyelination in the taiep rats. On the contrary, the increased CXCL1 mRNA and protein levels in other myelin mutants such as jimpy mice were associated with a remyelination process in the spinal cord [41]. However, CXCL1 mRNA was not affected in the taiep rats; the decrease of CXCL1 protein levels could be explained by instability of mRNA or a failure in its regulation. Related to the former suggestion, it has been reported that CXCL1 mRNAs contain adenine-uridine-rich sequence elements (AREs) in their $3^{\prime}$-untranslated regions ( $3^{\prime}$-UTRs) that exhibit constitutive instability. These regions are stabilized by acute proinflammatory stimulus, that is, IL-17 [42-44], that is not present in the taiep rats.

The 1-month-old taiep rat showed difference between mRNA and protein CXCR4 that might be due to two causes: (1) a decrease in stabilization of CXCR4 mRNA or an increase in CXCR4 protein translation that would rapidly consume CXCR4 mRNA [45] and (2) the increase of CXCR4 protein in the brainstem that could be due to the presence of other CXCR4 positive cells such as OPCs that are known to migrate from ventral ventricular zone during myelination, which is still active at one month of age [46]. Supporting the latter suggestion is the finding that mRNA and protein CXCR4 are decreased in the brainstem of 6-month-old taiep rats, when the migration of OPCs was completed [47].
We found that VEGF mRNA and protein levels are increased in 6-month-old taiep rats. This factor released by astrocytes is known to disrupt the blood-brain barrier in CNS inflammatory disease [48]. In addition, VEGF promotes OPC migration to the myelination areas [49]. However, OPC migration is altered in taiep rats $[7,8]$ despite the increased VEGF levels. The alteration of OPC migration might be explained by the deficiency of CXL1 and CXCR4 found here. The role of VEGF in OPCs migration was proven by blocking VEGF receptor with anti-Flk-1 antibody [49]. Finally, the increased levels of VEGF, CXCL10, and CCL19 might account for the infiltration of lymphocytes CD4+ and $\mathrm{CD} 8+$ previously found in the taiep rats at the age of 6 months.

\section{Conclusion}

The mutation in the taiep rats that causes the microtubule accumulation in the oligodendrocyte and the consequent hypomyelination is still unknown $[1,2,50]$. Our results strongly suggest that the deficiency of the remyelinationstimulating factors such as CXCL1 and CXCR4 contributes to the failure in the process of remyelination in a microenvironment of chronic inflammation in the taiep rats. Further studies are needed to identify signal pathways and other mediators involved in the inflammatory response of the taiep rat.

\section{Conflict of Interests}

The authors have no financial, personal, or other relationships with other people or organizations within five years of beginning the submitted work. The authors declare that they have no conflict of interests.

\section{Acknowledgments}

Victor Manuel Blanco-Alvarez, Constantino Tomas-Sanchez, and Guadalupe García-Robles were recipients of scholarships from CONACYT. This work was supported by VIEP (Grant NAT/2014). Thanks are due to Rasagna Nadella, Ph.D., for the editing of the English-language text.

\section{References}

[1] I. D. Duncan, K. F. Lunn, B. Holmgren, R. Urba-Holmgren, and L. Brignolo-Holmes, "The taiep rat: a myelin mutant with an associated oligodendrocyte microtubular defect," Journal of Neurocytology, vol. 21, no. 12, pp. 870-884, 1992.

[2] E. Couve, J. F. Cabello, J. Krsulovic, and M. Roncagliolo, "Binding of microtubules to transitional elements in oligodendrocytes of the myelin mutant taiep rat," Journal of Neuroscience Research, vol. 47, no. 6, pp. 573-581, 1997.

[3] B. A. León-Chávez, J. A. Gonzalez-Barrios, A. Ugarte, M. A. Meraz, and D. Martinez-Fong, "Evidence in vitro of glial cell priming in the taiep rat," Brain Research, vol. 965, no. 1-2, pp. 274-278, 2003.

[4] B. A. Leon-Chavez, P. Aguilar-Alonso, J. A. Gonzalez-Barrios et al., "Increased nitric oxide levels and nitric oxide synthase 
isoform expression in the cerebellum of the taiep rat during its severe demyelination stage," Brain Research, vol. 1121, no. 1, pp. 221-230, 2006.

[5] G. Soto-Rodríguez, D. Martinez-Fong, P. Aguilar-Alonso et al., "Nitric oxide production is associated to increased lipoperoxidation and active caspase- 3 in demyelinated brain regions of the taiep rat," Advances in Bioscience and Biotechnology, vol. 3, no. 6, pp. 695-704, 2012.

[6] A. Wilkins, Y. Kondo, J. Song et al., "Slowly progressive axonal degeneration in a rat model of chronic, nonimmune-mediated demyelination," Journal of Neuropathology and Experimental Neurology, vol. 69, no. 12, pp. 1256-1269, 2010.

[7] A. K. Foote and W. F. Blakemore, "Repopulation of oligodendrocyte progenitor cell-depleted tissue in a model of chronic demyelination," Neuropathology and Applied Neurobiology, vol. 31, no. 4, pp. 374-383, 2005.

[8] A. K. Foote and W. F. Blakemore, "Inflammation stimulates remyelination in areas of chronic demyelination," Brain, vol. 128, no. 3, pp. 528-539, 2005.

[9] B. A. L. Chavez, J. Guevara, S. Galindo et al., "Regional and temporal progression of reactive astrocytosis in the brain of the myelin mutant taiep rat," Brain Research, vol. 900, no. 1, pp. 152$155,2001$.

[10] J. S. Stoolman, P. C. Duncker, A. K. Huber, and B. M. Segal, "Site-specific chemokine expression regulates central nervous system inflammation and determines clinical phenotype in autoimmune encephalomyelitis," The Journal of Immunology, vol. 193, no. 2, pp. 564-570, 2014.

[11] B. C. Kieseier, M. Tani, D. Mahad et al., "Chemokines and chemokine receptors in inflammatory demyelinating neuropathies: a central role for IP-10," Brain, vol. 125, no. 4, pp. 823834, 2002.

[12] R. N. Aravalli, S. Hu, T. N. Rowen, J. M. Palmquist, and J. R. Lokensgard, "Cutting edge: TLR2-mediated proinflammatory cytokine and chemokine production by microglial cells in response to herpes simplex virus," Journal of Immunology, vol. 175, no. 7, pp. 4189-4193, 2005.

[13] M. J. Trifilo and T. E. Lane, "Adenovirus-mediated expression of CXCL10 in the central nervous system results in T-cell recruitment and limited neuropathology," Journal of NeuroVirology, vol. 9, no. 3, pp. 315-324, 2003.

[14] R. E. Kohler, I. Comerford, S. Townley, S. Haylock-Jacobs, I. Clark-Lewis, and S. R. McColl, "Antagonism of the chemokine receptors CXCR3 and CXCR4 reduces the pathology of experimental autoimmune encephalomyelitis," Brain Pathology, vol. 18, no. 4, pp. 504-516, 2008.

[15] D. W. Wojkowska, P. Szpakowski, D. Ksiazek-Winiarek, M. Leszczynski, and A. Glabinski, "Interactions between neutrophils, Th17 Cells, and chemokines during the initiation of experimental model of multiple sclerosis," Mediators of Inflammation, vol. 2014, Article ID 590409, 8 pages, 2014.

[16] E. Ambrosini, M. E. Remoli, E. Giacomini et al., "Astrocytes produce dendritic cell-attracting chemokines in vitro and in multiple sclerosis lesions," Journal of Neuropathology and Experimental Neurology, vol. 64, no. 8, pp. 706-715, 2005.

[17] S. Robinson, M. Tani, R. M. Stricter, R. M. Ransohoff, and R. H. Miller, "The chemokine growth-regulated oncogene-alpha promotes spinal cord oligodendrocyte precursor proliferation," The Journal of Neuroscience, vol. 18, no. 24, pp. 10457-10463, 1998.

[18] L. Kadi, R. Selvaraju, P. de Lys, A. E. I. Proudfoot, T. N. C. Wells, and U. Boschert, "Differential effects of chemokines on oligodendrocyte precursor proliferation and myelin formation in vitro," Journal of Neuroimmunology, vol. 174, no. 1-2, pp. 133146, 2006.

[19] K. M. Omari, G. R. John, S. C. Sealfon, and C. S. Raine, "CXC chemokine receptors on human oligodendrocytes: implications for multiple sclerosis," Brain, vol. 128, no. 5, pp. 1003-1015, 2005.

[20] H.-H. Tsai, E. Frost, V. To et al., "The chemokine receptor CXCR2 controls positioning of oligodendrocyte precursors in developing spinal cord by arresting their migration," Cell, vol. 110, no. 3, pp. 373-383, 2002.

[21] J. R. Patel, E. E. McCandless, D. Dorsey, and R. S. Klein, “CXCR4 promotes differentiation of oligodendrocyte progenitors and remyelination," Proceedings of the National Academy of Sciences of the United States of America, vol. 107, no. 24, pp. 11062-11067, 2010.

[22] R. Bonavia, A. Bajetto, S. Barbero, P. Pirani, T. Florio, and G. Schettini, "Chemokines and their receptors in the CNS: expression of CXCL12/SDF-1 and CXCR4 and their role in astrocyte proliferation," Toxicology Letters, vol. 139, no. 2-3, pp. 181-189, 2003.

[23] A. Bajetto, S. Barbero, R. Bonavia et al., "Stromal cell-derived factor- $1 \alpha$ induces astrocyte proliferation through the activation of extracellular signal-regulated kinases $1 / 2$ pathway," Journal of Neurochemistry, vol. 77, no. 5, pp. 1226-1236, 2001.

[24] A. Bajetto, R. Bonavia, S. Barbero, T. Florio, and G. Schettini, "Chemokines and their receptors in the central nervous system," Frontiers in Neuroendocrinology, vol. 22, no. 3, pp. 147-184, 2001.

[25] J. J. Sedmak and S. E. Grossberg, "A rapid, sensitive, and versatile assay for protein using coomassie brilliant blue G250," Analytical Biochemistry, vol. 79, no. 1-2, pp. 544-552, 1977.

[26] J. J. Dimatelis, N. S. Pillay, A. K. Mutyaba, V. A. Russell, W. M. U. Daniels, and D. J. Stein, "Early maternal separation leads to down-regulation of cytokine gene expression," Metabolic Brain Disease, vol. 27, no. 3, pp. 393-397, 2012.

[27] J. McQueen, M. M. Reimer, P. R. Holland et al., "Restoration of oligodendrocyte pools in a mouse model of chronic cerebral hypoperfusion," PLoS ONE, vol. 9, no. 2, Article ID e87227, 2014.

[28] A. Fratangeli, E. Parmigiani, M. Fumagalli et al., "The regulated expression, intracellular trafficking, and membrane recycling of the P2Y-like receptor GPR17 in Oli-neu oligodendroglial cells," Journal of Biological Chemistry, vol. 288, no. 7, pp. 5241-5256, 2013.

[29] M. Liu, J. Zhao, K. Chen et al., "G protein-coupled receptor FPR1 as a pharmacologic target in inflammation and human glioblastoma," International Immunopharmacology, vol. 14, no. 3, pp. 283-288, 2012.

[30] S. Columba-Cabezas, B. Serafini, E. Ambrosini, and F. Aloisi, "Lymphoid chemokines CCL19 and CCL21 are expressed in the central nervous system during experimental autoimmune encephalomyelitis: implications for the maintenance of chronic neuroinflammation," Brain Pathology, vol. 13, no. 1, pp. 38-51, 2003.

[31] M. Krumbholz, D. Theil, F. Steinmeyer et al., "CCL19 is constitutively expressed in the CNS, up-regulated in neuroinflammation, active and also inactive multiple sclerosis lesions," Journal of Neuroimmunology, vol. 190, no. 1-2, pp. 72-79, 2007.

[32] C. Trebst, S. M. Staugaitis, P. Kivisäkk et al., "CC chemokine receptor 8 in the central nervous system is associated with phagocytic macrophages," The American Journal of Pathology, vol. 162, no. 2, pp. 427-438, 2003.

[33] D. Y. Choi, M. K. Lee, and J. T. Hong, "Lack of CCR5 modifies glial phenotypes and population of the nigral dopaminergic 
neurons, but not MPTP-induced dopaminergic neurodegeneration," Neurobiology of Disease, vol. 49, no. 1, pp. 159-168, 2013.

[34] W. G. Glass, M. T. Liu, W. A. Kuziel, and T. E. Lane, "Reduced macrophage infiltration and demyelination in mice lacking the chemokine receptor CCR5 following infection with a neurotropic coronavirus," Virology, vol. 288, no. 1, pp. 8-17, 2001.

[35] H. Tokami, T. Ago, H. Sugimori et al., "RANTES has a potential to play a neuroprotective role in an autocrine/paracrine manner after ischemic stroke," Brain Research, vol. 1517, pp. 122-132, 2013.

[36] A. Salmaggi, M. Gelati, A. Dufour et al., "Expression and modulation of IFN- $\gamma$-inducible chemokines (IP-10, Mig, and I-TAC) in human brain endothelium and astrocytes: possible relevance for the immune invasion of the central nervous system and the pathogenesis of multiple sclerosis," Journal of Interferon \& Cytokine Research, vol. 22, no. 6, pp. 631-640, 2002.

[37] J. H. Dufour, M. Dziejman, M. T. Liu, J. H. Leung, T. E. Lane, and A. D. Luster, "IFN- $\gamma$-inducible protein 10 (IP-10; CXCL10)deficient mice reveal a role for IP-10 in effector T cell generation and trafficking," Journal of Immunology, vol. 168, no. 7, pp. 31953204, 2002.

[38] R. Filipovic and N. Zecevic, "The effect of CXCL1 on human fetal oligodendrocyte progenitor cells," Glia, vol. 56, no. 1, pp. $1-15,2008$.

[39] R. Filipovic, I. Jakovcevski, and N. Zecevic, "GRO-alpha and CXCR2 in the human fetal brain and multiple sclerosis lesions," Developmental Neuroscience, vol. 25, no. 2-4, pp. 279-290, 2003.

[40] I. Jakovcevski, R. Filipovic, Z. Mo, S. Rakic, and N. Zecevic, "Oligodendrocyte development and the onset of myelination in the human fetal brain," Frontiers in Neuroanatomy, vol. 3, article 5, 2009 .

[41] Q. Wu, R. H. Miller, R. M. Ransohoff, S. Robinson, J. Bu, and A. Nishiyama, "Elevated levels of the chemokine GRO-1 correlate with elevated oligodendrocyte progenitor proliferation in the Jimpy mutant," The Journal of Neuroscience, vol. 20, no. 7, pp. 2609-2617, 2000.

[42] S. Datta, M. Novotny, P. G. Pavicic Jr. et al., "IL-17 regulates CXCL1 mRNA stability via an AUUUA/tristetraprolin- independent sequence," Journal of Immunology, vol. 184, no. 3, pp. 1484-1491, 2010.

[43] T. Hamilton, M. Novotny, P. J. Pavicic et al., "Diversity in posttranscriptional control of neutrophil chemoattractant cytokine gene expression," Cytokine, vol. 52, no. 1-2, pp. 116-122, 2010.

[44] M. Novotny, S. Datta, R. Biswas, and T. Hamilton, "Functionally independent AU-rich sequence motifs regulate KC (CXCL1) mRNA," The Journal of Biological Chemistry, vol. 280, no. 34, pp. 30166-30174, 2005.

[45] N. Al-Souhibani, M. Al-Ghamdi, W. Al-Ahmadi, and K. S. A. Khabar, "Posttranscriptional control of the chemokine receptor CXCR4 expression in cancer cells," Carcinogenesis, vol. 35, no. 9, pp. 1983-1992, 2014.

[46] M. Bradl and H. Lassmann, "Oligodendrocytes: biology and pathology," Acta Neuropathologica, vol. 119, no. 1, pp. 37-53, 2010.

[47] N. Kessaris, M. Fogarty, P. Iannarelli, M. Grist, M. Wegner, and W. D. Richardson, "Competing waves of oligodendrocytes in the forebrain and postnatal elimination of an embryonic lineage," Nature Neuroscience, vol. 9, no. 2, pp. 173-179, 2006.

[48] A. T. Argaw, L. Asp, J. Zhang et al., "Astrocyte-derived VEGFA drives blood-brain barrier disruption in CNS inflammatory disease," The Journal of Clinical Investigation, vol. 122, no. 7, pp. 2454-2468, 2012.
[49] K. Hayakawa, L.-D. D. Pham, A. T. Som et al., "Vascular endothelial growth factor regulates the migration of oligodendrocyte precursor cells," Journal of Neuroscience, vol. 31, no. 29, pp. 10666-10670, 2011.

[50] K. F. Lunn, P. W. Baas, and I. D. Duncan, "Microtubule organization and stability in the oligodendrocyte," Journal of Neuroscience, vol. 17, no. 13, pp. 4921-4932, 1997. 


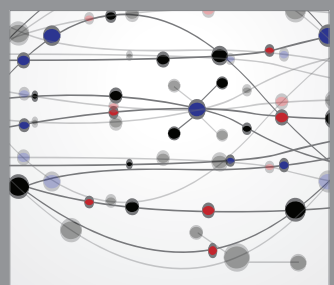

The Scientific World Journal
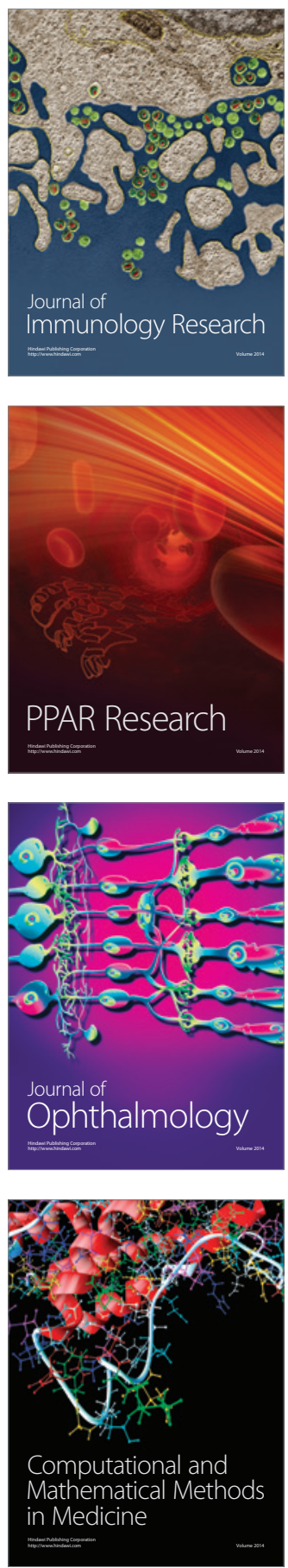

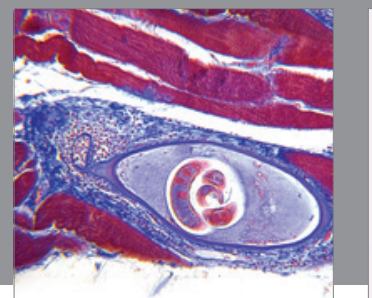

Gastroenterology

Research and Practice
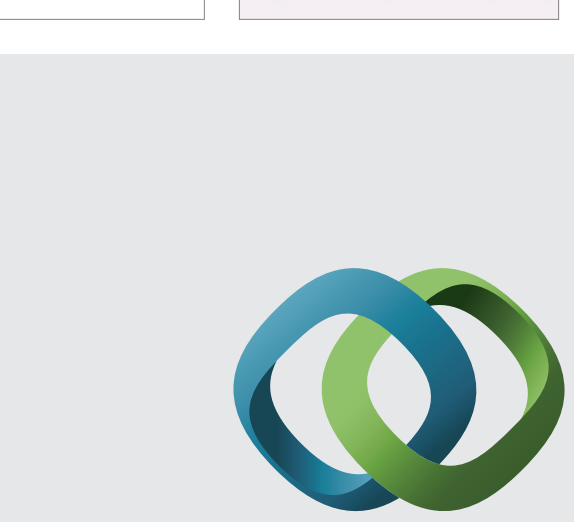

\section{Hindawi}

Submit your manuscripts at

http://www.hindawi.com
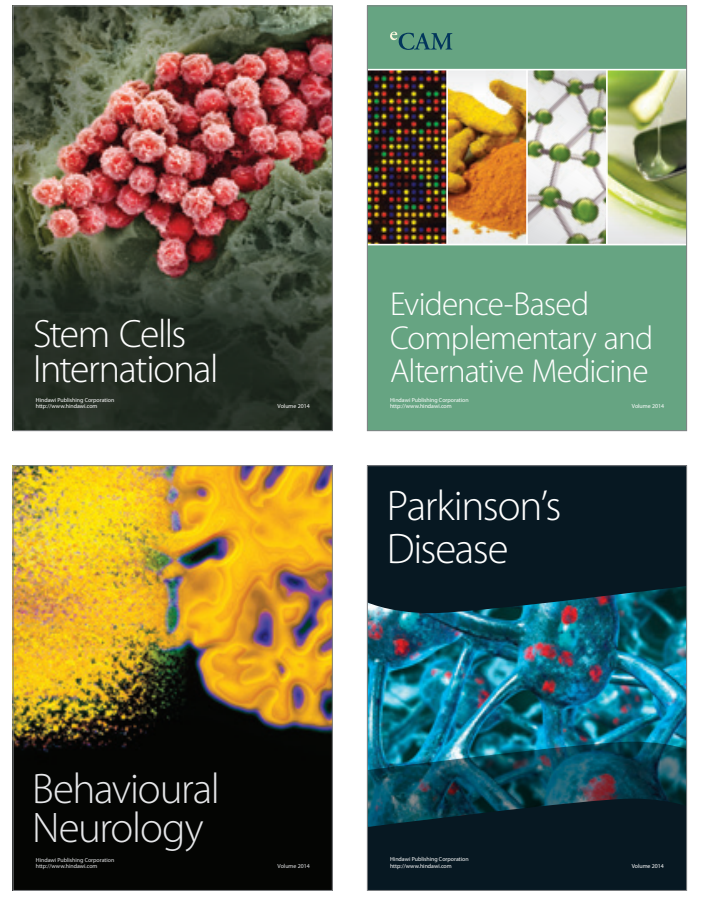
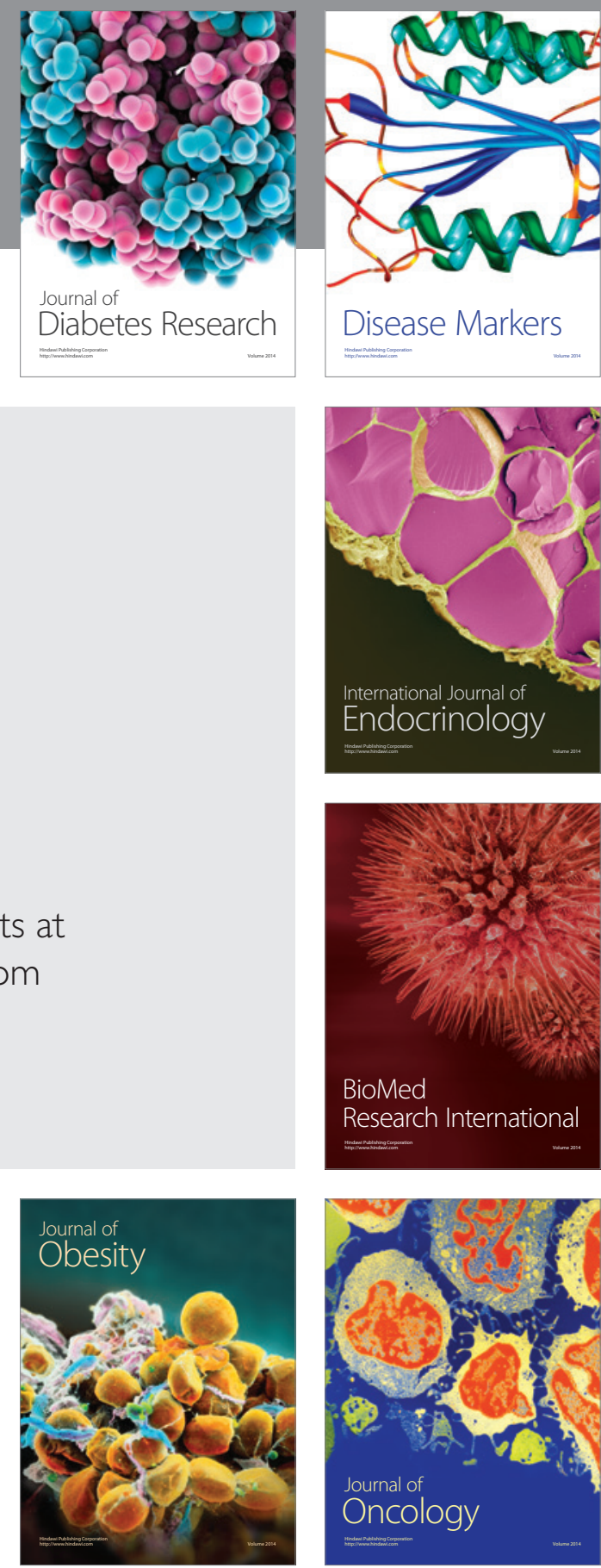

Disease Markers
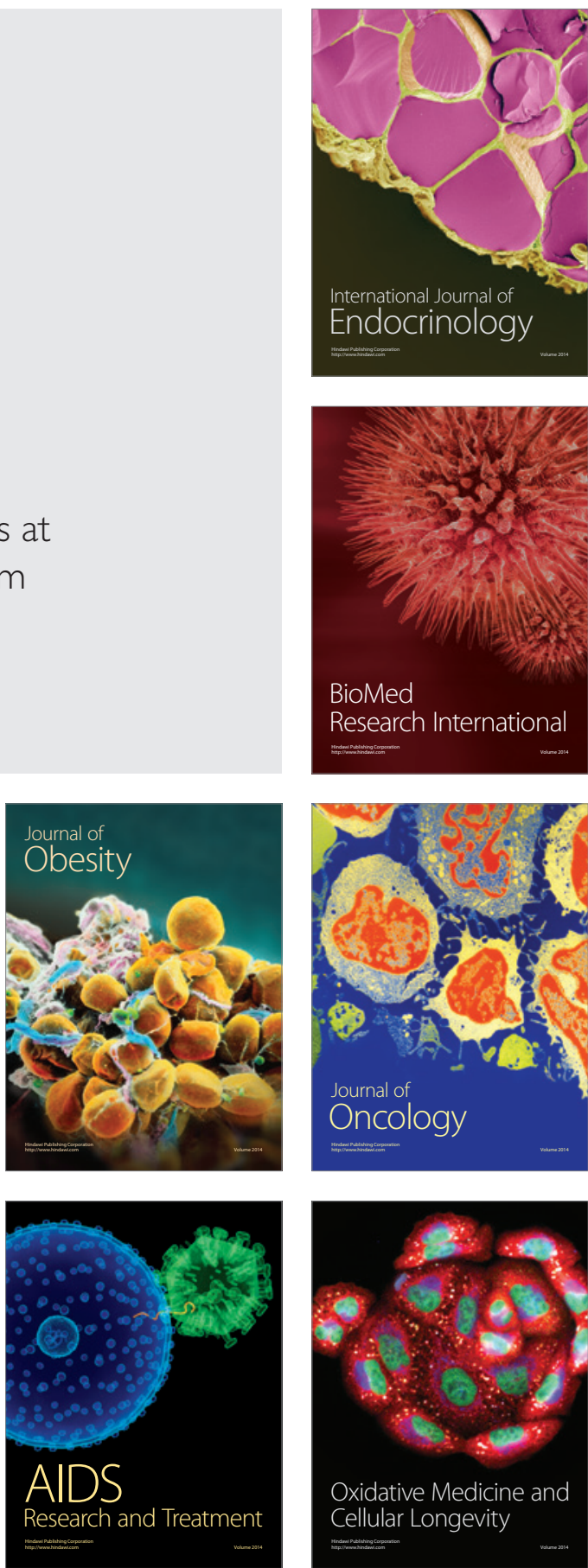